\title{
The process of interaction between abrasive disk and a workpiece while grinding under ultrasonic vibrations
}

\author{
Alexandr Unyanin ${ }^{1, *}$, and Alexandr $\mathrm{Khazov}^{1}$ \\ ${ }^{1}$ Ulyanovsk State Technical University, Ulyanovsk, Russia
}

\begin{abstract}
This research includes the analysis of kinematic relations between abrasive disk grains and the workpiece in the abrading process while applying ultrasonic vibrations (USV) on the workpiece. Supposition, that applying vibrations lied in axes of abrasive disk increases efficiency of disk grinding, has been set. Analytical approach showed dependences for calculating the grinding force. These dependences include changing of disk grains microcutting kinematics and mechanical characteristics of workpieces while applying USV. The technique of pilot studies is given. For imposing on preparation of USV used the device in which it was one of links of oscillatory system that provides the minimum losses of energy USV and high amplitude of fluctuations of preparation. Field researches have shown, that applying USV lied in axes of abrasive disk decreased the grinding force, the wear of disk, and high-level parameters of the processed surface roughness.
\end{abstract}

\section{Introduction}

Process of grinding of tough material workpieces has high intensity of wearing and loading during grinding of disk working surface; it also causes a high heating rate. This factor makes difficult to have high quality surface while high productive process. Using of external energy forces, e. g. ultrasonic vibrations is one of the ways to increase the efficiency of grinding process.

The main regularities of influence of ultrasonic fluctuations USV on processes of machining are revealed in works $/ 1-4 /$. However influence of USV on indicators of process of grinding was estimated generally experimentally.

A significant amount of works is directed to studying of kinematics of grinding, however only in the few the kinematics with imposing of USV is analyzed. In work $/ 5 /$ the

* Corresponding author: a un@mail.ru 
microcutting kinematics is considered by the single abrasive grains (AG), the scheme of a trajectory of the movement of abrasive grains concerning preparation is provided. The analytical dependences describing regularities of the movement AG concerning preparation when imposing USV aren't given. The lack of analytical researches complicates development of the recommendations concerning use of USV for increase in efficiency of process of grinding.

Research objective - development of the recommendations submitted at choice of the rational direction of fluctuations and their amplitude.

For achievement of goals the following problems were solved:

- analytical researches of kinematics of interaction of AG of a circle with preparation and grinding forces with imposing of USV;

- pilot studies of efficiency of process of grinding with imposing of USV;

- analysis of results and development of recommendations about application of USV.

\section{Analytical researches}

The result of the analytical research of kinematic relations between AG of grinding disk (GD) and the workpiece while applying USV in different directions is the following dependences for calculations: vibration frequency for changing the speed direction of grains in respect of the workpiece; coordinates of transitionfrom plastic deformation with abrasive grains to microcutting and back; the number of grains for dispersion of workpiece material in works $/ 6,7 /$.

Researches of kinematics give us the following conclusions:

- to increase the speed of AG in respect of the workpiece and decrease the frictional force while applying USV lied in $x$-axis, the over industrial standard vibrations are needed (Fig. 1);

- applying of vibrations lied in $y$-axis is needed to form vital height-level parameters of the surface's microgeometry (roughness);

- applying of vibrations lied in $z$-axis (as the abrasive disk rotates) gives minimal height-level parameters of roughness. In that case the workpiece vibration amplitude in normal direction is enough to decrease the frictional coefficient of AG. That is why it is more efficient to apply vibrations in this direction.

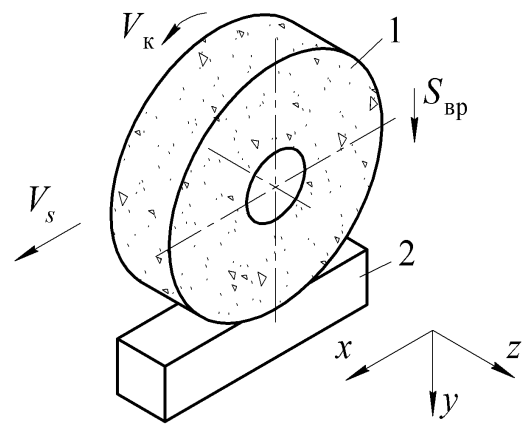

Fig. 1. Applying vibrations on the workpeace:

1 - grinding disk;

2 - workpeace 
Vibrations can be applied both on the GD and the workpiece. Applying on the workpiece is easier to process. So, all following calculations are made for applying USV lied in $z$-axis while processing flat grinding.

Components of the grinding force $P_{y}$ and $P_{z}$ are the sum of cutting and plastic deformation of AG. Each one is connected with material dispersing and dragging of AG with the workpiece:

$$
\begin{aligned}
& P_{y}=P_{y r 1}+P_{y r 2}+P_{y d 1}+P_{y d 2} ; \\
& P_{z}=P_{z r 1}+P_{z r 2}+P_{z d 1}+P_{z d 2},
\end{aligned}
$$

where $P_{y r 1}$ and $P_{z r 1}, P_{y r 2}$ and $P_{z r 2}$ - radial (suffixy) and tangential (z) components of the grinding force, accordingly connected to workpiece material microcutting (suffix 1 ) and dragging of cutting AG with the workpiece (suffix 2), N; $P_{y d 1}$ and $P_{z d 1}, P_{y d 2}$ and $P_{z d 2}-$ components of grinding force, accordingly connected with plastic deformation of workpiece material and plastic deformation of AG with the workpiece, $\mathrm{N}$.

Every component was calculated by assuming relevant grains forces which contact with the workpiece. The number of cutting and plastic deformation AGs depends on disk characteristics and the depth of penetration of grains in the workpiece material. The AG depth of penetration can be changed by changing the position of grains on the workpiece contact arc and also by changing the USV parameters (amplitude and frequency).

Cutting AGs are placed on the GD surface on the following distance $0 \leq y \leq y(l)$, where $y(l)$ - function, that describes depending the AG depth of penetration $y$ and the length $l$ of the contact with the workpiece.We can calculate the component $P_{y p 1}$ by using multiple integral while assuming microcutting forces with single grains.

$$
P_{y p 1}=\int_{0}^{l}\left[\int_{0}^{y(l)} P_{y r l i} \cdot n_{z k 1} \cdot c_{k} \cdot d y\right] \cdot d l,
$$

where $l_{k}$ - the length of contact act of GD with the workpiece, m; $P_{y r l i}$ - microcutting force with a single grain, $\mathrm{N} ; n_{z k 1}$ - the number of AG on the disk surface in its plane section parallel to disk axis, $1 / \mathrm{m}\left(n_{z k 1}=Z_{0} \cdot H\right.$, where $Z_{0}$ - the number of AG on the disk unit of area, $1 / \mathrm{m}^{2} ; H$ - size of the processing workpiece surface, parallel to the GD axis, $\mathrm{m}$ ); $c_{k}-$ coefficient, that is depended on GD characteristics /8/.

$$
y(l)=h_{u}+a_{z}(l)+A_{y} \cdot \sin \left(\omega \frac{l}{V_{K}}+\varphi\right),
$$

where $h_{u}$ - total value of grains chipping while shaping and the dimensional wear of GD, $\mathrm{m} ; a_{z}(l)$ - function, that describes changing of AG depth of penetration in the workpiece material on the contact length $l$ without applying USV; $A_{y}$ - workpiece amplitude of 
vibration, perpendicular to processed surface (lied inyaxis), $\mathrm{m} ; V_{K}-\mathrm{GD}$ working speed, $\mathrm{m} / \mathrm{s} ; \omega$ - vibrations circular frequency, $\mathrm{rad} / \mathrm{s} ; \varphi-$ USV phase shift, $\mathrm{rad}$.

Force of microcutting with a single grain:

$$
P_{y r 1 i}=\tau_{s} \cdot F_{m} \cdot k_{y}
$$

where $\tau_{s}$ - shear stress while microcutting the workpiece material with a grain, $\mathrm{Pa} ; F_{m}-$ area of the workpiece metal taken out with a single, $\mathrm{m}^{2} ; k_{y}$ - coefficient $/ 8 /$.

As a result of calculations, here is the following dependence:

$$
\begin{aligned}
& P_{y r 1}=k_{y} \cdot \tau_{s} \cdot \operatorname{tg} \gamma \cdot n_{z k 1} \cdot c_{k} \cdot\left(2 \cdot h_{u}{ }^{2} \cdot a_{k r} \cdot l_{k}+\left(6 \cdot h_{u} \cdot a_{k r}+7 \cdot h_{u}{ }^{2}+a_{k r}{ }^{2}\right) A \cdot \frac{l_{k}^{2}}{2}+h_{u} \cdot a_{k r}{ }^{2} \cdot l_{k}+\right. \\
& \left.+\left(h_{u}+a_{k r}\right) \cdot h_{u}^{2} \cdot l_{k}+\frac{h_{u}^{3} \cdot l_{k}}{3}+K_{1 U Z}\right),
\end{aligned}
$$

where $K_{1 U Z}$ - component, that shows changings of microcutting kinematics under USV; $a_{k r}$ - critical depth of microcutting - if this value is exceeded, the plastic deformation is changed to microcutting, $\mathrm{m} ; \gamma$ - half-value of the angle on the AG top, deg.; $l_{k}$ - length of AG contact arc with the workpiece, $m$.

$$
A=\frac{V_{s} \cdot \sin \alpha_{\max } \cdot l_{m}}{\pi \cdot D_{k} \cdot n_{k} \cdot l_{k}},
$$

where $l_{m}$ - average distance between cutting AGs, $\mathrm{m} ; V_{s}-$ traverse speed, $\mathrm{m} / \mathrm{min} ; D_{k}-$ diameter of the grinding disk, $\mathrm{m} ; n_{k}-$ revolution rate of the disk, $1 / \mathrm{min} ; \alpha_{\max }-$ angle to the length of AG contact arc to the workpiece, deg. /9, 10/.

The following equations show other components grinding force $P_{y}$.

$$
P_{y r 2}=\frac{\pi \cdot l_{2 r}^{2} \cdot k_{y} \cdot n_{z k 1} \cdot c_{k} \cdot \tau_{s}}{12 \cdot \mu_{s}} \cdot\left(h_{u} \cdot l_{k}+\frac{A \cdot l_{k}^{2}}{2}+K_{2 U Z}\right),
$$

where $l_{2 r}$ - size of cutting AG blunting area, $\mathrm{m} ; \mu_{s}$ - internal friction coefficient of processing material; $K_{2 U Z}$ - dependence component, that includes changing of microcutting kinematics while applying USV.

$$
P_{y d 1}=\frac{c \cdot \sigma_{t} \cdot n_{z k 1} \cdot c_{k} \cdot \operatorname{tg} \gamma}{2}\left(\sin \gamma+\mu_{0} \cdot \cos \gamma\right) \cdot l_{k} \cdot a_{k r} \cdot\left(\frac{a_{k r}^{2}}{3}-h_{u d}^{2}\right),
$$

where $c$ - coefficient; $\sigma_{t}$ - workpiece material yield stress, $\mathrm{Pa} ; h_{u d}$ - wear of AG, that complete the plastic deformation of workpiece material, $m ; \mu_{0}$ - friction coefficient of AG and workpiece. 


$$
P_{y d 2}=\frac{c \cdot \sigma_{t} \cdot k_{y} \cdot \pi \cdot l_{2 d}^{2} \cdot n_{z k} \cdot c_{k} \cdot a_{k r} \cdot l_{k}}{12}
$$

where $l_{2 d}$ - size of AG plastic deformation blunting area, $\mathrm{m}$.

$P_{y d 1}$ and $P_{y d 2}$ do not depend on ultrasonic vibration parameters.

The same way is applicable to calculate dependences of $P_{z}$ components.

Listed above dependences for calculating forces are applicable for low values of workpieces material particles vibration amplitude, perpendicular to processed surface. If the values of amplitude are low, than AG mode (microcutting or plastic deformation) stays unchanged.

Workpiece vibrations make the dispersion process of the material more efficient. Applying USV decreases stress limit $\sigma_{\mathrm{B}}$ and yielding $\sigma_{t}$ of workpiece material $/ 11 /$.

The results of analytical and field researches show that the difference of values of the force under the USV amplitude $A_{z} \leq 1,5$ мкм is no more than $14 \%$. Changing the microcutting kinematics with single grains under the low amplitude USV does not cause changing of grinding forces. They (forces) are decreased by decreasing a stress and yielding limit of workpiece material under USV.

Grinding forces of plastic deformation of grains $P_{y d 1}, P_{y d 2}, P_{z d 1}, P_{z d 2}$ are insignificant comparing with $P_{y}$ and $P_{z}$. The sum of material plastic deformation forces is not more than 6\% $P_{y}$ and $P_{z}$.

\section{Pilot studies}

Field researches were processed under flat pendular grinding by grinding disk face using $3 \mathrm{H} 3 \mathrm{M} 3 \mathrm{~F}$ $(3 \mathrm{X} 3 \mathrm{M} 3 \Phi)$ and $12 \mathrm{H} 18 \mathrm{~N} 10 \mathrm{~T}(12 \mathrm{X} 18 \mathrm{H} 10 \mathrm{~T})$ steels. These types of steel are in 1 and 3 group of grinding and have very high plastic properties. Grinding processes by disk 25A25(F60)ПСМ1 $(K) 6 \mathrm{~K} 6(V)$ with working speed $V_{K}=35 \mathrm{~m} / \mathrm{s}$, traverse speed $V_{s}=10 \mathrm{~m} / \mathrm{min}$; in feed motion $S_{\text {вр }}=0,01 \mathrm{~mm} /$ motions per act; USV frequency $f=18,6 \mathrm{kHz}$; USV amplitude, lied inzaxis-between 1,5 and $6 \mathrm{mkm}$. Cutting coolant - $3 \%$ calcined coda was poured to the grinding zone as fast as $10 \mathrm{dm}^{3} / \mathrm{min}$.

While applying vibrations a special tool was used. In that case the workpiece was a part of vibrating system (Fig. 2) /12/. Workpiece 4 is set into the nest 3 and fixed with support 8 to the front of USV gun 6 with screws 10 . The nest 3 is set to the magnetic plate 2 of the machine. Elastic mechanical vibrations of ultrasonic frequency, generated by piezoelectric converters 9 , go to the workpiece through the gun 6 set in the box 7. The total length of a gun, workpiece, and support must be the same as USV wave length, but with maximum amplitude frequency of the workpiece. Minimum energy loss and high amplitude frequency can be also achieved by well contacted gun and workpiece.

Such tools can be used in flat gridding machines to process hard-processing materials (highly alloyed steel, titan alloys), that cannot be set on the magnetic plate. 
To estimate the efficiency of grinding process the following values are used: $P_{y}$ and $P_{z}$; wear of grinding disk; grinding coefficient $K_{c h}$; redress life $\tau_{\mathrm{c}}$, min; parameters of grinding surface roughness $R a, R z$, Rmax, mkm.

$P_{y}$ and $P_{z}$ were calculated with dynamometric tool UDM-100. Its signal goes through signal booster to ACP 16/16 - SIGMA/USB using ZetLab Studio software.

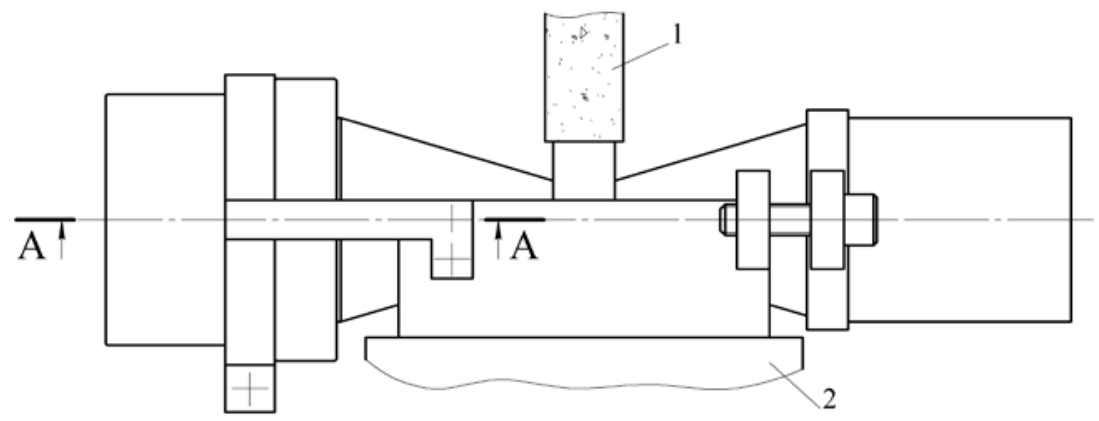

A - A

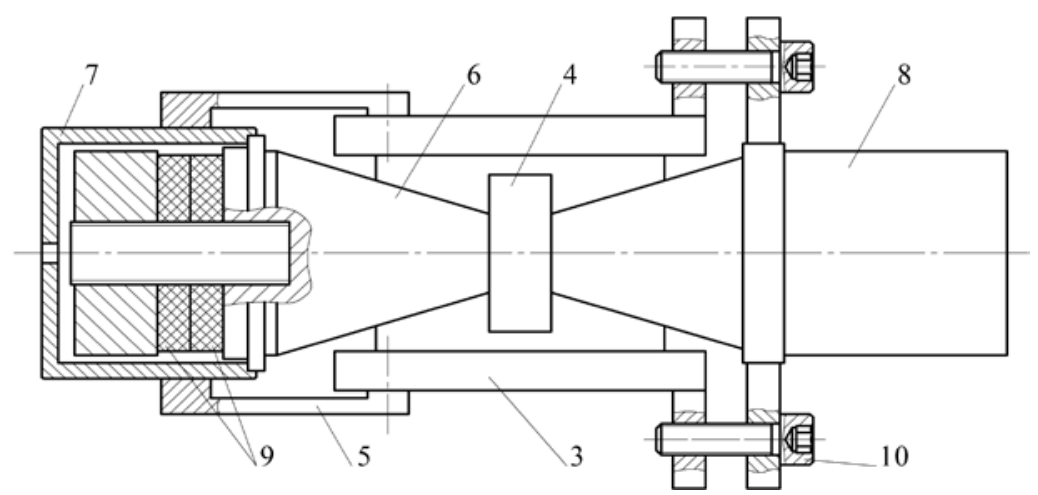

Fig. 2. Applying USV to the workpiece:

1 - GD; 2 - magnetic plate; 3 - nest; 4 - workpiece; 5 - support arm;

6 - gun; 7 - box; 8 - support; 9 - piezoelectric converters; 10 - screws

\section{Results of researches and their discussion}

As the workpiece amplitude frequency go high, so the components of grinding force go low. The minimum value of force was detected with $6 \mathrm{mkm}$ amplitude. $P_{y}$ and $P_{z}$ was decreased with such amplitude while processing 3 X3M3 $\Phi$ steel by $10 \%$ comparing to non USV (Table1). While processing 12H18X10T steel, $P_{y}$ and $P_{z}$ was decreased by на15 and $11 \%$ correspondingly. 
Table 1. Influence of USV amplitude on grinding force components

\begin{tabular}{|c|c|c|c|c|c|}
\hline \multirow{2}{*}{$\begin{array}{c}\text { Workpiece } \\
\text { material }\end{array}$} & \multicolumn{5}{|c|}{ Amplitude $A_{z}$ of USV, mkm } \\
\cline { 2 - 6 } & 0 & 1,5 & 3 & 4,5 & 6 \\
\hline $3 \mathrm{X} 3 \mathrm{M} \Phi$ & $15,2 / 13$ & $14,8 / 12,5$ & $14,2 / 12,3$ & $13,9 / 12,1$ & $13,6 / 11,7$ \\
\hline $12 \mathrm{X} 18 \mathrm{H} 10 \mathrm{~T}$ & $14,5 / 13,5$ & $13,6 / 12,8$ & $13 / 12,4$ & $12,8 / 12,4$ & $12,3 / 12$ \\
\hline \multicolumn{7}{|c|}{ Note. Numerator includes $P_{y}$, denominator includes $P_{z}$} \\
\hline
\end{tabular}

The minimal wear of the disk is detected while grinding $3 \mathrm{X} 3 \mathrm{M} 3 \Phi$ and $12 \mathrm{X} 18 \mathrm{H} 10 \mathrm{~T}$ types of steel applying USV amplitude 3 and 4.5 correspondingly. This wear is 42 and $24 \%$ less if USV is not used. After increasing amplitude frequency up to $6 \mathrm{mkm}$, the wear might increase because of shearing and grain smoothness.

The grinding coefficient for 3X3M3Ф and 12X18H10T steel was increased by 70 and $37 \%$ correspondingly. It can be explained as decreasing the disk wear after applying USV.

Redness life was increased in 3 to 3.5 times while applying USV.The redress life is increased by $3 . . .3,5$ times.

While grinding without USV, roughness parameters are increased with the time of operating grinding disk. It is very noticeable while grinding $3 \mathrm{X} 3 \mathrm{M} 3 \Phi$ steel. While applying USV on the workpiece lied in grinding disk axis, small parts of material vibrate perpendicular because of shear waves. Perpendicular to processed surface vibrations increase the number of grains with different height. That is why dependence of height parameters of roughness on USV amplitude is so vital.

The minimal value of height parameters of roughness is noticed with USV amplitude $A_{z}$ is $4,5 \mathrm{mkm}$. This amplitude decreased $R a$ parameter by 25 and $24 \%$ while grinding $3 \mathrm{X} 3 \mathrm{M} 3 \Phi$ and $12 \mathrm{X} 18 \mathrm{H} 10 \mathrm{~T}$ types of steel correspondingly. The same influence is on $R z$ and Rmax parameters.

\section{Main results and conclusions}

The analysis of kinematics of interaction of AG of a circle with preparation in the course of grinding with imposing on preparation of USV is made. It is established that it is expedient to impose fluctuations in the direction coinciding with an axis of a grinding wheel.

With analytical way dependences for calculation of forces of grin ding with imposing of USV are received and experimentally confirmed.

The result of analytical research is finding out dependences for calculating forces for flat grinding while applying USV. By an experimental approach was proved the fact that applying USV on the workpiece decreases the grinding force by $10 \ldots 15 \%$, disk wear by $25 \ldots 40 \%$, roughness height parameters by 25 $\%$; the grinding coefficient was increased up to $70 \%$, and the redress life by $3 \ldots 3.5$ times. 


\section{References}

1. Podurayev V.N. Processing by cutting with vibrations. (1970)

2. Vologin M.F., Kalashnikov V.V., Nerubay M.S., Shtrikov B.L. Application of ultrasound and explosion during the processing and assembly. (2002)

3. Baldev R., Radzhendran V., Palanichami Item. Application of ultrasound. (2006)

4. Agapov S.I., Golovkin V.V. Increase in efficiency of mechanical processing by application of ultrasound. (2010)

5. Tawakoli T. Industrial Diamond Review. (2009)

6. A.N. Unyanin, I.Y.Terekhin. Proceedings of the seventh international STC "Mathematical modeling of physical, economic, technical, social systems and processes". (2009)

7. A.N. Unyanin, Y.A. Arzamascev. Proceedings of the IV international scientific and technical conference. (2012)

8. Efimov V.V. Model of grinding process using coolant. (1992)

9. Hudobin L.V., Unjanin A.N. Izvestiyavuzov. Engineering. (2006)

10. Hudobin L.V., Unjanin A.N. To minimize the clogging of grinding wheels. (2007)

11. Shtrikov B.L., Malkina I.V. Materials of the international STC «High technologies in mechanical engineering». -SGTU. (2005)

12. A. N. Unyanin, I. Yu. Terekhin, Patent RU 2418671 RF, IPC B 24 B 1 / 04, B 24 B 5 / 04, 14 (2011) 